Check for updates

Cite this: RSC Adv., 2019, 9, 3469

Received 25th November 2018

Accepted 4th January 2019

DOI: $10.1039 / c 8 r a 09692 a$

rsc.li/rsc-advances

\section{Controlling the shear thickening behavior of suspensions by changing the surface properties of dispersed microspheres}

\begin{abstract}
Yi. Chen, (D) *ab Yueyun Zhou, ${ }^{a}$ Hejie $\mathrm{Pj}^{\mathrm{ab}}$ and Guangsheng Zeng*bc
To investigate the effect of the surface properties of dispersed particles on the shear thickening behavior of their corresponding suspensions and further control this characteristic, three kinds of suspensions were prepared by mixing $\mathrm{SiO}_{2}, \mathrm{SiO}_{2}-\mathrm{NH}_{2}$, and $\mathrm{SiO}_{2}-\mathrm{COOH}$ microspheres with a poly(ethylene glycol) fluid medium, and their rheological behavior was analyzed carefully. Compared to the $\mathrm{SiO}_{2} \mathrm{microsphere}$ suspension, the $\mathrm{SiO}_{2}-\mathrm{NH}_{2}$ and $\mathrm{SiO}_{2}-\mathrm{COOH}$ microsphere suspensions show a weaker thickening behavior and a greater critical shear rate due to the aggregation tendency caused primarily by the organic chains. Moreover, the rheological behavior of the three suspensions display different dependencies on the $\mathrm{pH}$ value, which is comprehensively determined by the interaction between the microspheres and the medium. Moreover, the critical shear stress of suspensions with different $\mathrm{pH}$ values could be predicted by the Wagner model, which basically proves that the interaction between the particles significantly influences the beginning of thickening. The thickening degree could be interpreted by friction theory. The critical volume fraction corresponding to the onset of discontinuous shear thickening is determined by the friction coefficient between the particles, which is greatly affected by the $\mathrm{pH}$ value.
\end{abstract}

\section{Introduction}

The rheology of dense suspensions is of significant theoretical and technological importance due to its great potential in energy absorption, physical protection, magnetic damping and so on. ${ }^{1-3}$ However, shear rheology of even the simplest case of a suspension composed of hard spheres and Newtonian fluid is incompletely understood. Based on different particle suspensions, many attempts were made to explain how shear thickening happens, and some important mechanisms were advanced successively. Typically, the order-disorder transition theory, linking shear thickening to the breaking of ordered alignment of the layered structure above a critical shear rate,,$^{4,5}$ the dilatancy theory, regarding the thickening as a tendency of granular materials to expand underflow, ${ }^{6,7}$ the "hydroclusters" theory, in which the thickening is ascribed to the formation of jamming clusters by hydrodynamic lubrication forces, ${ }^{\mathbf{8 , 9}}$ have

${ }^{a}$ Hunan Provincial Key Laboratory of Comprehensive Utilization of Agricultural and Animal Husbandry Waste Resources, College of Urban and Environmental Sciences, Hunan University of Technology, Zhuzhou, 412007, China. E-mail: yiyue514@ aliyun.com; 290427057@qq.com

${ }^{b}$ Hunan Provincial Engineering Laboratory of Key Technique of Non-metallic Packaging Waste Resources Utilization, Hunan University of Technology, Zhuzhou 412007, China. E-mail: guangsheng_zeng@126.com

${ }^{c}$ Hunan Provincial Key Laboratory of Biomass Fiber Functional Materials, Hunan University of Technology, Zhuzhou 412007, China been proven to be reasonable by experiments and simulations in special research systems.

In addition to mechanistic exploration, a more pertinent question is whether this kind of special rheological behavior can meet the demands of practical applications.

For example, in self-protection and magnetic damping systems, there are various application demands, including the kind of impact or shear that needs to be resisted, the intensity of the magnetic field leading to the shear thickening, the degree of thickening for suitable energy absorption and so on.

In a colloidal suspension, the shape, size and surface structure of the particles and the viscosity and chemical structure of the media impact the rheological behavior significantly. ${ }^{10,11}$ However, alteration of the composition and material of the suspensions generally leads to an uncontrollable change of rheological behavior and other properties; thus, adjusting the thickening behavior in a controllable and simple method remains a great challenge. In shear thickening behavior, the most important feature is when the thickening happens and to what degree the thickening occurs, both of which are considered as important control objectives.

Focusing on the onset of thickening, analysis of the different mechanisms and prediction models related to the critical shear rate or critical shear stress was built up with a focus on different suspension systems. Based on hard sphere suspensions, including the mutual repulsion between the particles, Melrose and Ball established a model to predict the critical shear rate by 
hypothesizing that the time required for the conservative forces to separate a particle pair is equal to the time scale of the flow when shear thickening occurs. ${ }^{12}$ By modifying the model, Wagner and Maranzano established a new model to predict the critical shear stress of hard sphere suspensions by considering the particles size, concentration, surface potential and ionic strength. ${ }^{\mathbf{1 3 , 1 4}}$ Based on the electrically-stabilized colloidal suspensions in which the micro-interactions such as the van der Waals attraction is non-negligible. Kaldasch et al. established the model for predicting the critical stress by using DLVO theory to analyze the two-particle interaction. Moreover, they proved that sterically stabilized colloidal suspensions such as polymeric brush coating colloidal particles could also adopt this activation model. ${ }^{15,16}$ Obviously, in these models, the balance between the applied stress and the interaction of the particles determines the onset of shear thickening.

For the degree of thickening, in contrast to continuous shear-thickening (CST) which is a continuous viscosity increase at any applied rate, discontinuous shear-thickening (DST) is an order of magnitude jump in the stationary stress when the strain rate crosses some threshold value. This CST to DST transition is observed when the volume fraction of the flowing suspension increases above a critical value near the random close packing, which depends on the system properties, e.g. polydispersity or shape, and on the flow geometry. DST is a kind of thickening form which could show a better effect for energy absorption and physical protection. Some theoretical approaches have attempted to understand this abrupt viscosity increase. Holmes et al. suggested that DST could be interpreted as a shear-induced glass transition based on an ad hoc modecoupling theory. ${ }^{17-19}$ Brown gave the mechanism explanation that DST is related to the existence of an underlying jamming transition due to the frustration of the granular-like dilatancy by the confining stress. ${ }^{20-22}$ More recently, friction leading to the DST became the mainstream explanation of this phenomenon, that is, at low shear stress, the neighbouring particles are separated by a gap filled with solvent and interacts via hydrodynamic forces, which leads to a CST. While, at high shear stress, the repulsive force between the particles is overcome and thereby results in a frictional contact network and shear thickening behavior. ${ }^{23-26}$ Many other theoretical and experimental attempts were carried out to understand this effect. For example, new granular-like models where the additional frictional forces act between particles have been established to reproduce DST; frictional contact dynamics are expected to dominate especially at large volume fractions, close to the jamming transition, and is responsible for DST. ${ }^{27,28}$ Seto et al. introduced a numerical scheme including both relevant hydrodynamic interactions and granular-like contact, a critical shear stress associated with a critical shear rate where DST occurs, which separated the frictionless state and the frictional shear jammed state. ${ }^{29}$ Fernandez et al. identify a local Sommerfeld number that determines the transition from Newtonian to shear-thickening flows, and then showed that the suspension's volume fraction and the boundary lubrication friction coefficient controls the CST or DST transition. ${ }^{30}$ Wyart's assumptions about the contact proliferation predict two distinct types of DST in the absence of inertia. ${ }^{31}$ Comprehensively, the CST and DST behavior could be adjusted by altering the friction character of the particles.

Based on the foundation of previous research, it could be concluded that altering the short-range interactions can adjust the characteristics of shear thickening significantly. Though there have been many methods to change the shear thickening behavior of the suspensions, altering the interaction between components is considered as a simple and controllable approach. Applying an extra electric or magnetic field as an exogenous force to change the particle surface interactions has proved effective to control the rheological behavior of suspensions. $^{32,33}$ Similarly, the endogenous force appearing inside the systems should also work to cause the same effect. In this research, we tried to introduce different groups into the surface of the $\mathrm{SiO}_{2}$ microsphere so as to alter the surface characteristics of the microspheres and adjust the interaction inside the systems. Taking into account the electron donor and electron acceptor characteristic of the introduced groups, an aqueous solution with different $\mathrm{pH}$ values were added into the suspensions to modulate the interaction further due to the inductive and ionization effects. All these changes should relate to the onset and the degree of thickening. By doing this, it is hoped that shear thickening behavior could be controlled more effectively, and the effects of particle interactions on the thickening process could be understood in depth.

\section{Experimental}

\subsection{Materials}

The $\mathrm{SiO}_{2}$ microsphere used here was prepared by the stöber solgel method in our lab, ${ }^{34}$ the radius of the microsphere is about $120 \pm 10 \mathrm{~nm}$, as shown in Fig. 1a, the density is $2.24 \mathrm{~g} \mathrm{~cm}^{-3} \cdot \gamma-$ aminopropyl-triethoxysilane, methylbenzene, butanedioic anhydride, dimethylformamide was supplied by Aladdin Co., Ltd, China, polyethylene glycol with molecular weights of $200 \mathrm{~g}$
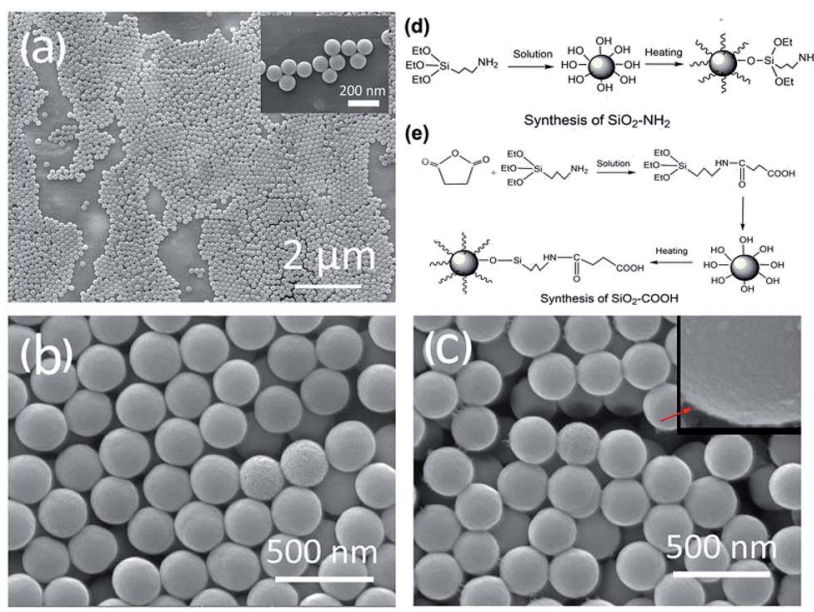

Fig. 1 The SEM images and modification scheme of the microsphere. (a) - (c) is the images of the prepared $\mathrm{SiO}_{2}, \mathrm{SiO}_{2}-\mathrm{NH}_{2}$ and $\mathrm{SiO}_{2}-\mathrm{COOH}$ microspheres. (d) and (e) is the modification scheme of the $\mathrm{SiO}_{2}-\mathrm{NH}_{2}$ and $\mathrm{SiO}_{2}-\mathrm{COOH}$ microspheres. 
$\mathrm{mol}^{-1}$ (PEG200) on average was supplied by Sigma-Aldrich Chemical GmbH, Düsseldorf, Germany, and is a low viscosity Newtonian media having, under $25^{\circ} \mathrm{C}$, a viscosity of $25 \mathrm{mPa} \mathrm{s}^{-1}$ and a density of $1.13 \mathrm{~g} \mathrm{~cm}^{-3}$.

\subsection{The synthesis of $\mathrm{SiO}_{2}-\mathrm{NH}_{2}$ microspheres}

The $\mathrm{SiO}_{2}-\mathrm{NH}_{2}$ microspheres were prepared by surface modification of the $\mathrm{SiO}_{2}$ microspheres, the scheme is shown in Fig. 1d. To describe it briefly, the prepared $\mathrm{SiO}_{2}$ particles were vacuum dried at $110^{\circ} \mathrm{C}$ for $5 \mathrm{~h}$ as an activation process, the activated $\mathrm{SiO}_{2}$ particles and methylbenzene solution with $5 \%$ wt $\gamma$ aminopropyl-triethoxysilane were added into a three-necked flask, the suspensions were heated to $110{ }^{\circ} \mathrm{C}$ and kept $8 \mathrm{~h}$ with magnetic stirring and reflux for reaction. Throughout the experiments, oxygen was eliminated from the system. After the reaction, the suspensions were extraction filtrated with a buchner funnel, the final modified $\mathrm{SiO}_{2}$ particles product was vacuum dried at $110{ }^{\circ} \mathrm{C}$ for $5 \mathrm{~h}$ and preserved in the vacuum drying oven. The grafting ratio of $-\mathrm{NH}_{2}$ could be adjusted by controlling the mass ratio of $\gamma$-aminopropyltriethoxysilane and the $\mathrm{SiO}_{2}$ particles. In this research, the content of $\mathrm{NH}_{2}$ in the surface of the $\mathrm{SiO}_{2}$ microsphere is $0.416 \mathrm{mmol} \mathrm{g}^{-1}$, which is measured by chemical titration. The size of the modified microsphere is basically equal to that of the unmodified microsphere, as shown in Fig. $1 \mathrm{~b}$.

\subsection{The synthesis of $\mathrm{SiO}_{2}-\mathrm{COOH}$ microspheres}

The scheme of $\mathrm{SiO}_{2}-\mathrm{COOH}$ microspheres preparation is shown in Fig. 1e. To briefly describe, equimolar $\gamma$-aminopropyltriethoxysilane and butanedioic anhydride were dispersed into dimethylformamide in a flask to form a $20 \mathrm{wt} \%$ solution, the solution was heated to $80^{\circ} \mathrm{C}$ for $3 \mathrm{~h}$ with magnetic stirring and reacted under reflux. Then, the homogeneous dispersed $\mathrm{SiO}_{2} /$ dimethylformamide suspension and a small quantity of deionized water were added into the system and stirred sequentially at $80{ }^{\circ} \mathrm{C}$ for $5 \mathrm{~h}$. The solid modified $\mathrm{SiO}_{2}$ particles were separated using ultracentrifugation, the final $\mathrm{SiO}_{2}-\mathrm{COOH}$ microspheres were obtained by washing several times with ethanol and centrifugation. In this research, the content of $\mathrm{COOH}$ in the surface of $\mathrm{SiO}_{2}$ microsphere is $0.402 \mathrm{mmol} \mathrm{g}{ }^{-1}$, which is measured by chemical titration. The size of the modified microsphere is equal to that of the unmodified microsphere basically, as shown in Fig. 1c.

\subsection{Preparation of suspension samples}

The suspensions were prepared by adding fully dried $\mathrm{SiO}_{2}$ and modified $\mathrm{SiO}_{2}$ microspheres into the liquid medium in a blender and mixing for $2 \mathrm{~h}$ in order to obtain a uniform distribution of particles within the suspension. The samples were sonicated for an hour and then placed in a vacuum chamber for $30 \mathrm{~min}$ to remove air bubbles and ensure that the particles were not aggregated. Afterwards, the suspensions were dispersed for $40 \mathrm{~min}$ by a superfine homogenizer (F6/10, Fluko Equipment Germany) at high speed (10 $000 \mathrm{rpm})$. The suspensions were allowed to rest for $12 \mathrm{~h}$ in an air tight plastic bottle, the final high concentration suspensions of $\mathrm{SiO}_{2}$ and modified $\mathrm{SiO}_{2}$ microspheres in PEG200 with various volume fractions were prepared. In this research, the volume fraction was calculated by the mass and density of the dispersed particles and the media, the density of the modified $\mathrm{SiO}_{2}$ microspheres is considered the same as the unmodified $\mathrm{SiO}_{2}$ microspheres. When the effect of the $\mathrm{pH}$ value on the rheological behavior was investigated, a small amount of aqueous solution (diluted solutions of $\mathrm{HCl}$ or $\mathrm{NaOH}$ ) with various $\mathrm{pH}$ values was added into the suspensions by a ratio of $1: 15$ to $1: 25$ in weight to that of the media.

\subsection{Friction measurements}

The friction between the microspheres cannot be directly and accurately measured due to their size and complex shape, a similar system was designed and measured by an universal micro-tribotester to simulate the actual situation. In test, a quartz sphere with a radius of $6 \mathrm{~mm}$ slides on a quartz disk in rotational mode was used to investigate the frictional properties between the microsphere surface. The rotational speed of the quartz disk was $100 \mathrm{rpm}$, and the radius of the track was $15 \mathrm{~mm}$. Throughout the tests, the load was kept constant at $1 \mathrm{~N}$. For simulating the real microspheres in suspensions, the special treatment was carried out in all test materials. For simulating the $\mathrm{SiO}_{2}$ microsphere, both the sphere and disk were treated using a piranha solution ( $7: 3$ concentrated $\mathrm{H}_{2} \mathrm{SO}_{4} / 30 \% \mathrm{H}_{2} \mathrm{O}_{2}$ ) for $20 \mathrm{~min}$ to form -OH groups on the surface as that of the $\mathrm{SiO}_{2}$ microsphere. In order to simulate the $\mathrm{SiO}_{2}-\mathrm{NH}_{2}$ and $\mathrm{SiO}_{2}-$ $\mathrm{COOH}$ microspheres further, the mixed solution A (methylbenzene solution with $5 \mathrm{wt} \% \gamma$-aminopropyl-triethoxysilane) and mixed solution B (dimethylformamide solution with $20 \mathrm{wt} \%$ equimolar $\gamma$-aminopropyl-triethoxysilane and butanedioic anhydride, reacting at $80{ }^{\circ} \mathrm{C}$ for $5 \mathrm{~h}$ ), as the reaction solutions used in surface modification of the microsphere, was coated on the surface of the piranha solution treated sphere and disk respectively. After that, the coated sphere and disk were kept at $80^{\circ} \mathrm{C}$ for $5 \mathrm{~h}$ and then vacuum dried for $2 \mathrm{~h}$. Through the treatment, the thickness of the surface covering layer of the sphere and disk reached $0.2-0.5 \mathrm{~mm}$ (the scheme is shown in Fig. 2). When testing the friction of the microspheres in $q$ special medium, the corresponding medium was dropped on the surface of the disk and spheres to wrap the test interface for a better simulation of the real situation.

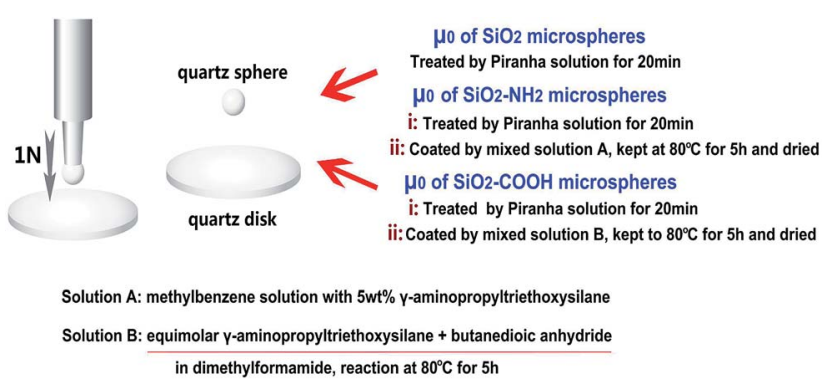

Fig. 2 The schematic representation for measuring the frictional properties of different microspheres. 


\subsection{Rheological tests}

Steady-shear rheological experiments on the suspensions were conducted using a rheometer TA Instruments ARES-G2 rheometer (supplied by TA. Instruments, USA). To prevent adhesive slip between the sample and the rheometer plates, parallel plates with a $25 \mathrm{~mm}$ diameter were covered with emery cloth (NORTON, K224) using double sided tape. The gaps explored were fixed at $0.8 \mathrm{~mm}$. Before the rheological test, a steady pre-shear was applied in the sample at a shear rate of $0.1 \mathrm{~s}^{-1}$ for $60 \mathrm{~s}$ in order to erase the shear history and an equilibrium time of $120 \mathrm{~s}$ was given at each shear rate to allow the system to reach a steady state. All the experiments were repeated at least three times.

\section{Results and discussion}

\subsection{The effect of surface property of microspheres on the shear thickening behavior}

In colloidal suspensions, the properties of the dispersed particles including size, shape, and softness have a significant effect on their rheological behavior. Especially, in some systems with small colloidal particles, the surface chemical structure of the particles is also important for the shear thickening due to the relatively stronger interaction between the particles. Based on this, the nano-sized $\mathrm{SiO}_{2}$ microsphere and its surface modified products, the $\mathrm{SiO}_{2}-\mathrm{NH}_{2}$ and $\mathrm{SiO}_{2}-\mathrm{COOH}$ microspheres were used to investigate the effect of the surface properties on shear thickening by comparing their rheological behavior.

Fig. 3 shows the shear rate versus the apparent viscosity curves of the suspensions. As shown in figure, for the different suspensions with the same volume fraction $(\phi)$, although the curves are approximately similar, the important features including the critical shear rate $(\dot{\gamma})$ defined as the shear rate at which the viscosity starts to increase significantly from the original decreasing or unchanged status, and the maximum viscosity still exhibits obvious differences, revealing the nonnegligible effects of the surface groups. The detailed $\dot{\gamma}$ data is listed in Fig. 3 b, from which it could be seen that, the rule $\dot{\gamma} \mathrm{SiO}_{2}$ $<\dot{\gamma} \mathrm{SiO}_{2}-\mathrm{NH}_{2}<\dot{\gamma} \mathrm{SiO}_{2}-\mathrm{COOH}$ is tenable for suspensions with the same $\phi$, the gap between them becomes greater at high $\phi$. It could be concluded that the grafted surface chains lead to a stronger occurrence of shear thickening, especially for suspensions with a high $\phi$.
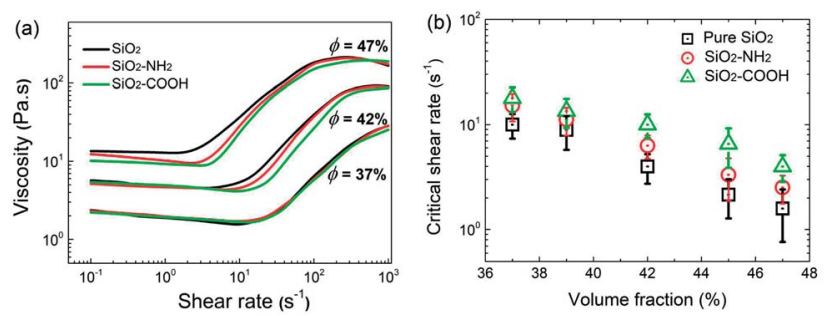

Fig. 3 The shear rate versus viscosity curves of three different suspensions with various volume fractions and the corresponding critical shear rate.

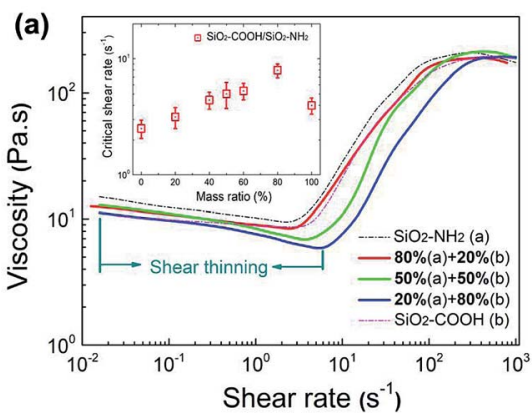

(b)

Fig. 4 The rheological behavior and micrograph of suspensions with mixed microspheres. (a) is the shear rate versus viscosity curves of suspensions with mixed microspheres. (b) and (c) is the SEM photo of pure $\mathrm{SiO}_{2}-\mathrm{NH}_{2}$ microspheres and mixed microspheres with $20 \%$ mass of $\mathrm{SiO}_{2}-\mathrm{NH}_{2}$ microspheres and $80 \%$ mass of $\mathrm{SiO}_{2}-\mathrm{COOH}$ microspheres.

Furthermore, the suspensions with mixed $\mathrm{SiO}_{2}-\mathrm{NH}_{2}$ and $\mathrm{SiO}_{2}-\mathrm{COOH}$ particles were prepared and their rheological curves are shown in Fig. 4. As shown in the figure, with an increased ratio of $\mathrm{SiO}_{2}-\mathrm{COOH}$ particles to the mixed particles, the $\dot{\gamma}$ of the suspensions increases significantly, and the first shear thinning becomes stronger, when the ratio of the $\mathrm{SiO}_{2}-$ $\mathrm{COOH}$ particles passes $50 \%$ of the total mass, the $\dot{\gamma}$ is greater than that of the pure $\mathrm{SiO}_{2}-\mathrm{COOH}$ particle suspensions, and the maximum viscosity decreases considerably, revealing a weaker shear thickening behavior.

Obviously, the surface grafted groups influence $\dot{\gamma}$ of the suspensions and leads to the significantly harder jamming. This change caused by the surface modification was analyzed. First, according to previous research, due to the amount of hydroxyls between the $\mathrm{SiO}_{2}$ particles and the dispersion media, a special solvation layer formed by hydrogen bonding appears near the surface, where the density of the oligomeric chains is higher than that of the bulk. This high density solvation layer is helpful to prevent the media fluid from flowing through the gap between the two spheres and acts as a "barrier", which promotes an earlier onset of shear thickening. ${ }^{35}$ Moreover, the thickness of this "strongly anchored" solvation layer could be approximately estimated. For $\mathrm{SiO}_{2}$ suspensions with a water and ethylene glycol (EG) media, the formed solvation layer thickness is $4.4 \mathrm{~nm}$ and $1.5 \mathrm{~nm}$, respectively. ${ }^{36,37}$ Taking account the similarity, the solvation layer of $\mathrm{SiO}_{2}$ microsphere in PEG is on the order of $1 \mathrm{~nm}$. While, after modification, the macromolecule chains cover the surface of the sphere and inhibit the formation of a solvation layer due to the destruction of the hydrogen bonding combination. However, this effect may be not large in this system due to the relatively small thickness of the solvation layer to the radius of the sphere $(120 \mathrm{~nm})$. Moreover, in the modified microspheres, the introduced groups both include low polarity organic chains, the similar polarity should lead to the slight aggregation of the microspheres and thereby result in a higher critical shear rate. This reason was proved further by the rheological behavior of the suspensions with the mixed particles.

In details, it was found that $\dot{\gamma} \mathrm{SiO}_{2}-\mathrm{COOH}$ is always higher than $\dot{\gamma} \mathrm{SiO}_{2}-\mathrm{NH}_{2}$, and is not a coincidence. Though both surface 
grafted chains have organic groups, the grafted chains in the $\mathrm{SiO}_{2}-\mathrm{COOH}$ microsphere is longer than that of the $\mathrm{SiO}_{2}-\mathrm{NH}_{2}$ microsphere, leading to a denser surface layer. Moreover, the interaction between the grafted chains themselves and between the grafted chains and media also has a significant effect on the shear thickening behavior, which will be discussed in the following section.

Another significant difference is the initial rheological behavior under a low shear rate. An obvious shear thinning was observed in the mixed particles suspensions, meaning a kind of relaxation or disentanglement behavior. In the modified microsphere, the introduced groups include a low polarity chain and a polar end group with an electron donor or electron acceptor, the aggregation should be intensified by charge attraction, and could be observed in the SEM images of the freeze dried $\left(-80^{\circ} \mathrm{C}\right)$ suspensions, by which the original status of the particles was basically maintained. As shown in Fig. 4, in comparison to the pure $\mathrm{SiO}_{2}-\mathrm{NH}_{2}$ particles, the mixed particles show micro-aggregation, the uniformity of the dispersion decreases. Moreover, the mixing ratio of the particles corresponding to the greatest $\dot{\gamma}$ and strongest shear thinning is consistent, which is $\mathrm{SiO}_{2}-\mathrm{COOH}: \mathrm{SiO}_{2}-\mathrm{NH}_{2}=8: 2$, instead of 1-to-1. This special ratio may be related to the highest aggregation degree, which is determined by the ratio and the nature of the introduced groups.

\subsection{The effects of $\mathrm{pH}$ value on the shear thickening behavior of suspensions}

The introduced grafted chains have a tendency for ionization in aqueous solutions due to their $\mathrm{NH}_{2}$ and $\mathrm{COOH}$ end groups, and therefore their interaction could be adjusted by altering the $\mathrm{pH}$ value. For this goal, a small amount of aqueous solution (5\% mass of suspensions) with different $\mathrm{pH}$ values were added into the suspensions. It is noteworthy that the actual $\mathrm{pH}$ of the suspensions is not consistent with that of the added solution, the measured $\mathrm{pH}$ value of the dilute silica microspheres aqueous dispersion without any addition is about 5.84, the neutralization effect decreases the acidity and alkalinity of the solution in a small range. For a convenient comparison, the discussed $\mathrm{pH}$ value in the following analysis is that of the added aqueous solution instead of the actual value of the suspensions. The viscosity of the media changes from $25 \mathrm{mPa}$ to $21.6 \mathrm{mPa} \mathrm{s}^{-1}$ after adding solution to whatever the $\mathrm{pH}$ value of the solution. The shear rate versus viscosity curves of the suspensions with different $\mathrm{pH}$ values are shown in Fig. 5. As shown in the figure, when the $\mathrm{pH}$ value changes, the rheological character of the suspensions including the $\dot{\gamma}$, the maximum viscosity, the thinning and thickening degree exhibits different changes.

A similar tendency was found in curves for the $\mathrm{SiO}_{2}$ and $\mathrm{SiO}_{2}-\mathrm{NH}_{2}$ suspensions, that is, with the increase of $\mathrm{pH}$, the $\dot{\gamma}$ and maximum viscosity increases considerably and the initial thinning and followed thickening becomes stronger. While, for the $\mathrm{SiO}_{2}-\mathrm{COOH}$ suspensions, the effect is reversed.

The reason for the diverse effects could be attributed to which one in the added solution is inclined to combine, the medium or the microsphere. This is determined by the
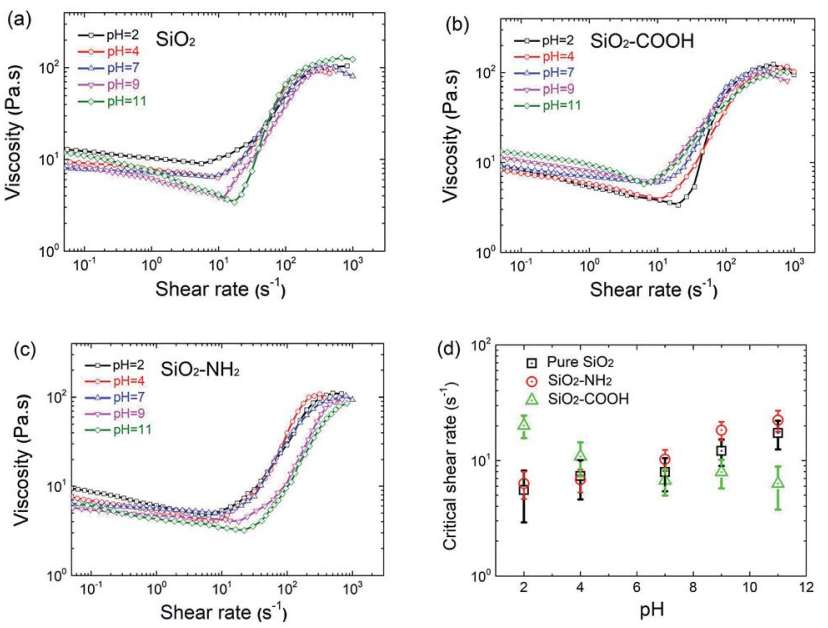

Fig. 5 The shear rate versus viscosity curves of three kind of suspensions with different $\mathrm{pH}$ values and their corresponding critical shear rate. (a) $\mathrm{SiO}_{2}$ suspensions; (b) $\mathrm{SiO}_{2}-\mathrm{COOH}$ suspensions; (c) $\mathrm{SiO}_{2}-\mathrm{NH}_{2}$ suspensions; (d) the critical shear rate of suspensions.

interaction comparison. When the added solution is inclined to combine with the media, the thinning becomes more significant, which is ascribed to the added aqueous solution working as a lubricant, the $\dot{\gamma}$ shifts to higher rate due to the decreased total viscosity, the thickening becomes more abrupt because of the lower effect of the added solution. When the added solution is inclined to combine with the microsphere, the result reverses.

The selective combination of the added solution may also be explained by the alkalinity or acidity of the materials in the system. In the $\mathrm{SiO}_{2}$ and $\mathrm{SiO}_{2}-\mathrm{NH}_{2}$ suspensions, $\mathrm{SiO}_{2}$ and PEG show weak acidity due to the $-\mathrm{OH}$ groups, the $\mathrm{SiO}_{2}-\mathrm{NH}_{2}$ particles exhibit a weak alkalinity due to the dissociation of the $-\mathrm{NH}_{2}$ groups in aqueous solution. When an alkali solution was added into the suspensions, it was easier for it to combine with PEG than the microspheres. As well, the microspheres adsorbed less solution which leads to a slight effect on the degree of shear thickening. When adding an acid solution, more solution is combined with the alkaline microspheres which brings about a weaker shear thickening and nearly unchanged shear thinning behavior. In the $\mathrm{SiO}_{2}-\mathrm{COOH}$ suspensions, the particles show a relatively stronger acidity in the aqueous solution than that of the $\mathrm{SiO}_{2}$ microsphere, the rule with regards to the selective combination becomes reversed to that of the $\mathrm{SiO}_{2}$ and $\mathrm{SiO}_{2}-\mathrm{NH}_{2}$ suspensions and thereby results in a different rheological behavior.

Based on the above results, it was found that the rheological behavior of the suspensions could be adjusted by changing their $\mathrm{pH}$ value. The key point we considered is the combined ability of the aqueous solution with the microspheres. For proving this point further, the mass of the added aqueous solution was adjusted in order to observe the change of the rheological behavior in detail. A significant distinction was found in the degree of thinning and thickening.

Taking a typical example, three masses of alkali solution(1/ $25,1 / 20$ and 1/15 mass of suspensions) were added into the $\mathrm{SiO}_{2}-\mathrm{C} \mathrm{OOH}$ suspensions, respectively, the viscosity of the pure 

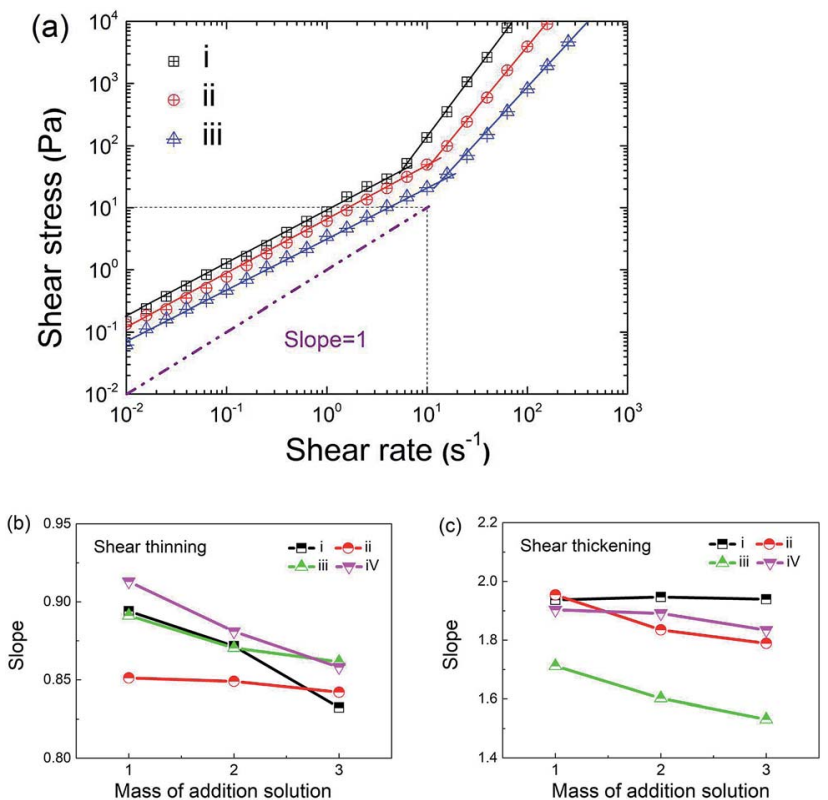

Fig. 6 The influence of adding aqueous solution with different $\mathrm{pH}$ value on the rheological behavior of suspensions. (a) The shear rate versus shear stress of $\mathrm{SiO}_{2}-\mathrm{COOH}$ suspensions with different added mass of aqueous solution ( $\mathrm{pH}=4$ ). (i)-(iii) corresponds to that the mass of added aqueous solution is $1 / 25,1 / 20$ and $1 / 15$ of suspensions. The volume fraction of suspensions is $47 \%$. (b) and (c) is the slope of suspensions (shear stress/shear rate) in the shear thinning region and shear thickening region of the suspensions with different adding mass of aqueous solution. In image (b) and (c), (i): adding acidic aqueous solution into $(\mathrm{pH}=4) \mathrm{SiO}_{2}-\mathrm{COOH}$ suspensions; (ii): adding alkali aqueous solution ( $\mathrm{pH}=11$ ) into $\mathrm{SiO}_{2}-\mathrm{COOH}$ suspensions; (iii): adding acidic aqueous solution ( $\mathrm{pH}=4$ ) into $\mathrm{SiO}_{2}-\mathrm{NH}_{2}$ suspensions; (iv): adding alkali aqueous solution $(\mathrm{pH}=11)$ into $\mathrm{SiO}_{2}-\mathrm{NH}_{2}$ suspensions, the abscissa 1, 2, 3 represents that the mass of addition solution is $1 / 25$, $1 / 20$ and $1 / 15$ of that of suspensions respectively.

media changes to $23.2,21.6$, and $20.2 \mathrm{mPa} \mathrm{s}^{-1}$, respectively, regardless of what the $\mathrm{pH}$ value of the solution is. The shear rate versus shear stress curves is exhibited in Fig. 6a for a more direct observation. As shown in the figure, the effect of added solution mass on the line slope (shear stress/shear rate) was different in the shear thinning and shear thickening region, the slope in the shear thinning region is basically unchanged with the change of the solution mass, while the slope in shear thickening region increases with the decreasing mass of added solution. The greater effect occurring in the shear thickening region proves that the alkali solution tends to combine more with the $\mathrm{SiO}_{2}-$ $\mathrm{COOH}$ microspheres than the media. Moreover, the same test was carried out in other kinds of suspensions, the slope in the shear thinning and thickening regions is shown in Fig. 6b and c, When the solution is easier to combine with media (such as adding an acidic solution into the $\mathrm{SiO}_{2}-\mathrm{COOH}$ suspensions or adding an alkali solution into $\mathrm{SiO}_{2}-\mathrm{NH}_{2}$ suspensions), the shear thinning becomes stronger and the shear thickening degree becomes weaker, when the solution is easier to combine with the microspheres (such as adding alkali solution into the $\mathrm{SiO}_{2}-$ $\mathrm{COOH}$ suspensions or adding acidic solution into $\mathrm{SiO}_{2}-\mathrm{NH}_{2}$ suspensions), the phenomenon is the inverse. From the slope change, the analysis above with respect to the selective combination could be demonstrated further.

We also note that, for the $\mathrm{SiO}_{2}$ suspensions, increasing the added aqueous solution always brings about a considerable decrease of the slope in the shear thickening region regardless of what the $\mathrm{pH}$ value of the added solution is, meaning that the $\mathrm{SiO}_{2}$ microspheres have a good ability to combine with the aqueous solution due to the existence of hydroxyls. By contrast, the surface grafted chains play the role of a hydrophobe to some degree.

\subsection{The mechanism analysis of the rheological behavior affected by $\mathrm{pH}$ value}

In the above analysis, it was found that the rheological behavior of the suspensions, especially its two main features, the beginning and the degree of thickening, could be influenced significantly by the changing the conditions due to the altered interaction between the particles. To figure out its mechanism, we tried to use the Wagner model which is suitable in hard sphere systems to predict the start of shear thickening. In this model, the critical shear stress $\left(\tau_{\mathrm{c}}\right)$ could be calculated by the surface potential and the radius of the particles, which is expressed by the following equation:

$$
\tau_{\mathrm{c}}=0.024 \frac{k_{\mathrm{B}} T(\kappa a) \Psi^{2}}{a^{2} l_{\mathrm{b}}}
$$

where $l_{\mathrm{b}}$ is the Bjerrum length defined by $l_{\mathrm{b}} \equiv e^{2} /\left(4 \pi \varepsilon \varepsilon_{0} k_{\mathrm{B}} T\right), a$ is the radius of the particle, and $\Psi=\psi_{\mathrm{s}} e / k_{\mathrm{B}} T$ is the dimensionless surface potential, $(\kappa a)$ is the relative double layer thickness. $\kappa^{-1}$ is the Debye length, which could be calculated by $\mathrm{hm}=1.453$ / $\kappa \cdot{ }^{38} \mathrm{hm}$ is the characteristic separation distance in the incipient hydrocluster state.

In this research, the media, the size of the particles and other conditions of the three suspensions is similar, the main impact on $\tau_{\mathrm{c}}$ could be ascribed to the changed interaction between the particles. As we know, the zeta potential of the particles could represent the dimensionless surface potential to some degree. Using this substitution, the $\tau_{\mathrm{c}}$ and zeta potential of the particles in different $\mathrm{pH}$ condition were measured and is shown in Fig. 7. It was found that the absolute value of the zeta potential exhibits a similar changing tendency as that of $\tau_{\mathrm{c}}$. For the $\mathrm{SiO}_{2}$ and $\mathrm{SiO}_{2}-\mathrm{COOH}$ suspensions, the $\tau_{\mathrm{c}}$ increases with the increase of $\mathrm{pH}$ value. For the $\mathrm{SiO}_{2}-\mathrm{NH}_{2}$ suspensions, the $\tau_{\mathrm{c}}$ decreases monotonously. Moreover, for the suspensions with the mixed particles, the same correlation between them was also found, which proves that the $\tau_{\mathrm{c}}$ closely links with the zeta potential of particles. The zeta potential could represent the stability of the particles in the system, regardless of whether it is the $\mathrm{SiO}_{2}$ or modified $\mathrm{SiO}_{2}$ microsphere, higher stability between the particles brings about higher $\tau_{\mathrm{c}}$.

Moreover, the actual value and predicted value of $\tau_{\mathrm{c}}$ calculated by using the Wagner equation are listed in Table 1, though the change tendency between the actual value and the predicted value is consistent, a discrepancy in the value still exists. Basically, the actual value is lower than the predicted value. For $\mathrm{SiO}_{2}$ microspheres, the effect of the solvation layer on the surface of 

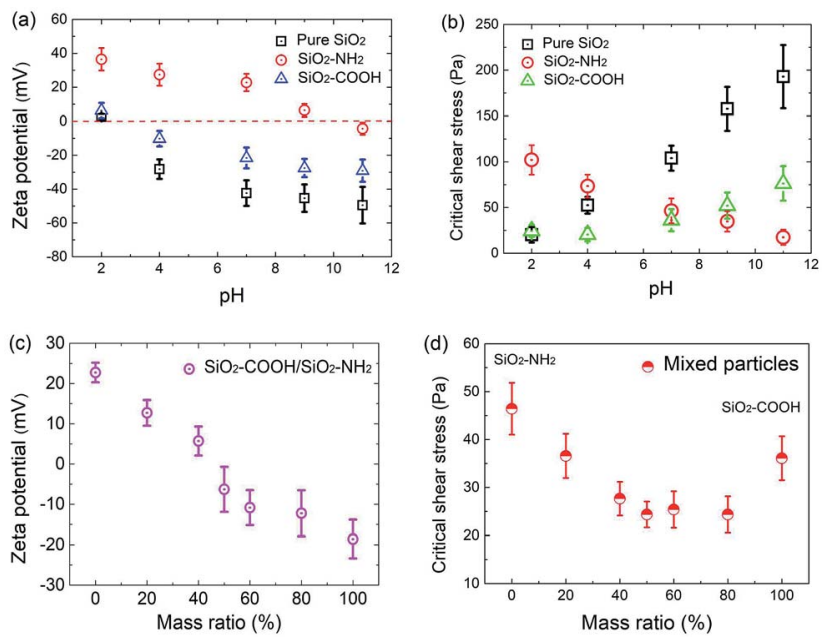

Fig. 7 The measured zeta potential of the particles in aqueous solution and their corresponding suspensions' critical shear stress. (a) the zeta potential of three kind particles in aqueous solution; (b) the critical shear stress of $\mathrm{SiO}_{2}, \mathrm{SiO}_{2}-\mathrm{NH}_{2}$ and $\mathrm{SiO}_{2}-\mathrm{COOH}$ suspensions; (c) the zeta potential of mixed particles with $\mathrm{SiO}_{2}-\mathrm{NH}_{2}$ and $\mathrm{SiO}_{2}-\mathrm{COOH}$; (d) the critical shear stress of mixed particles suspensions.

the microspheres may be ignored in theoretical calculation, the existence of a solvation layer leads to the decreased separation distance between microspheres, which makes the thickening happen more easily under lower stress. For the modified microspheres, there exist low-polarity surface organic groups, these groups interact with each other or interact with the molecular chains of the media, this kind of extra force is not included in the theoretical model.

CST and DST, linked to the different viscosity increase degree, both appear in this research system. The intrinsic factor determining the kind of shear thickening is a meaningful problem. Here, we tried to analyze it by using theory based on the friction coefficient between the particles.

Until now, it has been commonly accepted that the lubrication status between the particles was separated into two typical regimes which are determined by a characteristic number, the Sommerfeld number $s\left(s=\eta_{\mathrm{f}} \nu R_{\mathrm{p}} / N\right)$, where $\eta_{\mathrm{f}}$ is the fluid viscosity, $\nu$ is the sliding speed between the two solid surfaces, $R_{\mathrm{p}}$ is the radius of the spheres and $\mathrm{N}$ is the normal load. For high $s$, corresponding to the rheological status called the "hydrodynamic lubrication" regime (HD regime), the two sliding surfaces are fully separated by a fluid film and the friction coefficient $(\mu)$ depends on $s$. For low $s$, a "boundary lubrication" regime (BL regime) appears, in which the lubrication film breaks down and contacts between the microscopic asperities on each surface support most of the load, and $\mu$ exhibits only very weak dependency on $s$. The boundary of $s$ is about the order of $10^{-5}$. In some flow status, the "mixed regime" with both HD and BL also exists.

In the $\mathrm{HD}$ regime, the jamming volume fraction $\left(\phi \mathrm{HD}_{\max }\right)$ is at a random close packing $\phi \mathrm{RCP}$, regardless of the boundary friction coefficient. Conversely, the jamming volume fraction in the BL regime $\left(\phi \mathrm{BL}_{\max }\right)$ changes with changed $\mu$. When $\phi \leq$ $\phi \mathrm{BL}_{\max }$, the suspension exhibits CST, and when $\phi \mathrm{BL}_{\max }<\phi \leq$ $\phi \mathrm{HD}_{\max }$, the system undergoes DST. As a consequence, $\phi \mathrm{BL}_{\max }$ is the critical volume fraction for DST and therefore it can be tuned by changing the particle friction coefficient.

In this research, the shear thickening behavior of suspensions undergoes a transition from CST to DST with an increase of the volume fraction. This corresponding " $s$ " calculated by using the physical parameter of the materials and the rheological character $\left(R_{\mathrm{p}}=120 \mathrm{~nm}, \eta_{\mathrm{f}}=25 \mathrm{mPa} \mathrm{s}^{-1}, \nu\right.$ could be substituted by the shear rate, which is between $0.1-100 \mathrm{~s}^{-1}$ corresponding to the shear thickening region, the corresponding $\mathrm{N}$ measured by rheometer is $0.01-1 \mathrm{~N}$ ) is in the range of $10^{-7}$ to $10^{-8}$. We could reasonably consider that the systems are in boundary lubrication. Accordingly, the boundary lubrication between two rough particles could be described using the Amontons-Coulomb friction, the friction coefficient $\mu_{0}$ being independent of the load, speed and apparent contact area, corresponds to a special critical volume fraction that happens in DST. Here, the $\mu_{0}$ of the particles under different $\mathrm{pH}$ conditions was measured by using a special method, the value is shown in Fig. 8a. As shown in the figure, the $\mathrm{pH}$ value has a significant influence on the $\mu_{0}$ of the particles. The $\mu_{0}$ of the $\mathrm{SiO}_{2}$ and $\mathrm{SiO}_{2}-\mathrm{COOH}$ microspheres increases considerably with an increase of the $\mathrm{pH}$ value, while the $\mu_{0}$ of the $\mathrm{SiO}_{2}-\mathrm{NH}_{2}$ decreases. Obviously, the increased dissociation degree of the end groups advances $\mu_{0}$ between the particles. Fig. 8bd provides several actual examples with regard to how suspensions with the special particle $\mu_{0}$ changes from CST to DST $(n \geq$ 2, $n$ is Oswald-De Waele power law: $\tau=K \gamma^{n}$, when $n$ is higher than 2 , the thickening is discontinuous) with the increased volume fraction and the critical volume fraction $\left(\phi \mathrm{BL}_{\max }\right)$.

It is worth noting that the measured $\mu_{0}$ exhibits some correlation with the test load and speed. The test load affects $\mu_{0}$

Table 1 The actual and predicted critical stress of suspensions with different pH solution and the mixed particles suspensions ${ }^{a}$

\begin{tabular}{|c|c|c|c|c|c|c|c|c|c|}
\hline \multirow{2}{*}{$\mathrm{pH}$} & \multicolumn{2}{|l|}{$\mathrm{SiO}_{2}$} & \multicolumn{2}{|c|}{$\mathrm{SiO}_{2}-\mathrm{COOH}$} & \multicolumn{2}{|c|}{$\mathrm{SiO}_{2}-\mathrm{NH}_{2}$} & \multicolumn{3}{|l|}{ Mixed particles } \\
\hline & $A-v$ & $\mathrm{P}-\mathrm{v}$ & $A-v$ & $\mathrm{P}-\mathrm{v}$ & $A-v$ & $\mathrm{P}-\mathrm{v}$ & $\mathrm{MR}(\mathrm{COOH}: \mathrm{OH})$ & $A-v$ & $\mathrm{P}-\mathrm{v}$ \\
\hline 4 & 52.5 & 131.8 & 20.4 & 17.3 & 73.4 & 123.8 & $60: 40$ & 27.7 & 26.7 \\
\hline 7 & 104.1 & 296.8 & 36.1 & 77.2 & 46.4 & 85.5 & $50: 50$ & 24.4 & 5.4 \\
\hline 9 & 157.8 & 340.3 & 52.1 & 125.6 & 34.8 & 6.7 & $40: 60$ & 25.4 & 6.6 \\
\hline
\end{tabular}

${ }^{a}$ A-v is actual measured value, P-v is the predicted value, MR is the mass ratio. 

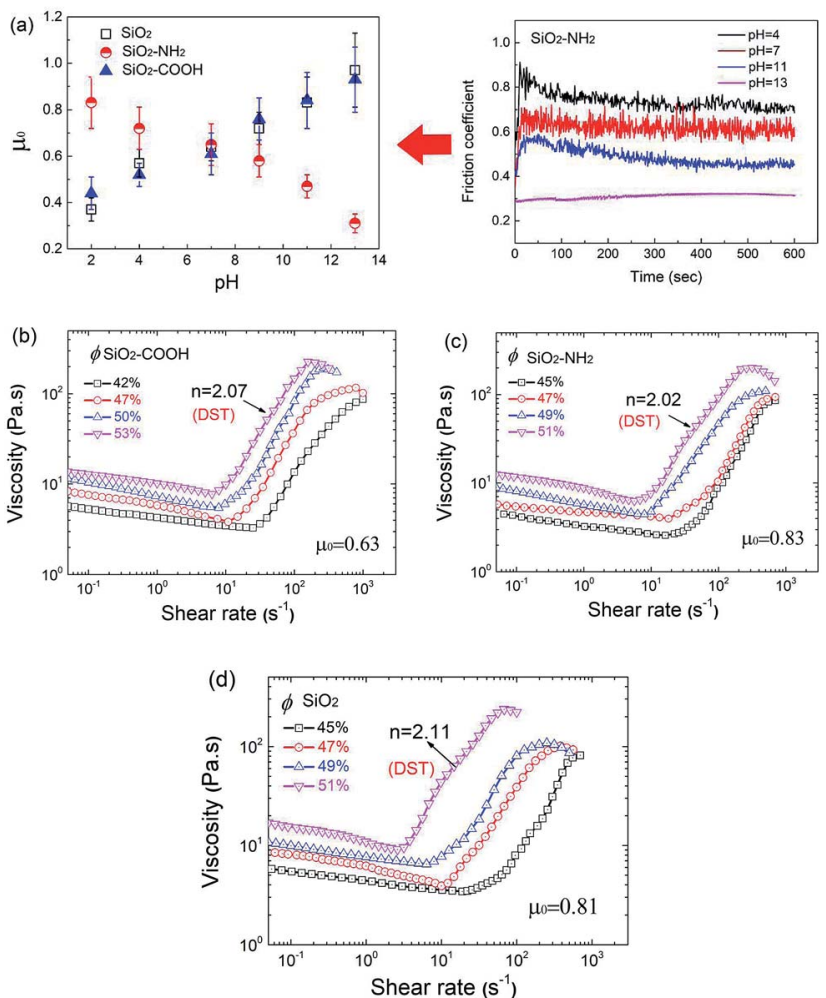

Fig. 8 The measured $\mu_{0}$ of particles under the different $\mathrm{pH}$ condition and the shear rate versus viscosity curve of three suspensions with special $\mu_{0}$. (a) The $\mu_{0}$ of particles under the different $\mathrm{pH}$ condition and its typical measured curves; (b) $\mathrm{SiO}_{2}-\mathrm{COOH}$ suspensions at $\mathrm{pH}=9$, the measured $\mu_{0}=0.63$; (c) $\mathrm{SiO}_{2}-\mathrm{NH}_{2}$ suspensions at $\mathrm{pH}=2$, the measured $\mu_{0}=0.83$; (d) $\mathrm{SiO}_{2}$ suspensions at $\mathrm{pH}=11$, the measured $\mu_{0}$ $=0.81, n$ is Oswald-De Waele power law: $\tau=K \gamma^{n}$.

more significantly, the test with the higher load could get a higher $\mu_{0}$. The test speed only has a slight effect on it. However, under the same test conditions, the relative magnitude of $\mu_{0}$ belonging to different suspensions is unchanged. Therefore, the test value could be used in qualitative analysis.

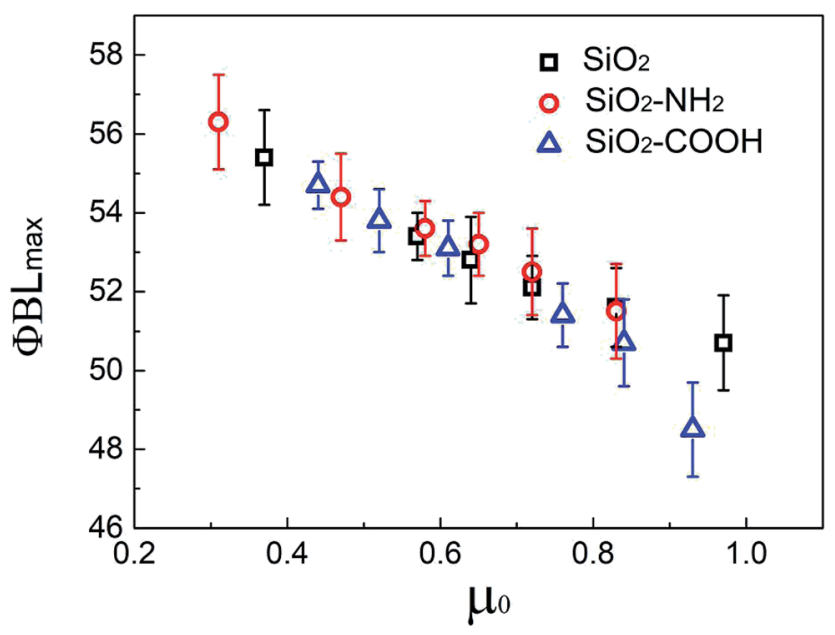

Fig. 9 The measured $\mu_{0}$ of three kinds of suspensions $(\phi=53 \%)$ at various $\mathrm{pH}$ conditions and their $\phi \mathrm{BL}_{\max }$ corresponding to the happen of DST.
The $\mu_{0}$ and corresponding $\phi \mathrm{BL}_{\max }$ for all suspensions with different $\mathrm{pH}$ value is shown in Fig. 9. Obviously, the $\phi \mathrm{BL}_{\max }$ decreases gradually with the increase of $\mu_{0}$, the $\phi \mathrm{BL}_{\max }$ for the same $\mu_{0}$ is similar even for different suspensions regardless of the surface chemical structure of the microsphere. It was proved further that the thickening behavior could be adjusted by changing the friction character of the particles, changing the $\mathrm{pH}$ value is a possible method for particles with polar or ionizable groups.

In order to quantify $\phi \mathrm{BL}_{\max }$, the viscosity versus shear rate curves in the thickening regime for different suspensions were fitted by the Oswald-De Waele power law: $\tau \propto \gamma^{n}$. It was found that the relationship of $n$ and $\phi$ is nonlinear. A uniform expression obtained using $\phi_{\max }$ corresponds to a strong thickening $(n=4)$ for three kinds of suspensions $(\mathrm{pH}=7)$ as a standard, the deviation of $\phi$ from the standard was normalized using $\left(\phi_{\max }-\phi\right) / \phi_{\max }$. As shown in Fig. 10a, the $n$ of the different suspensions collapse onto a single master curve as
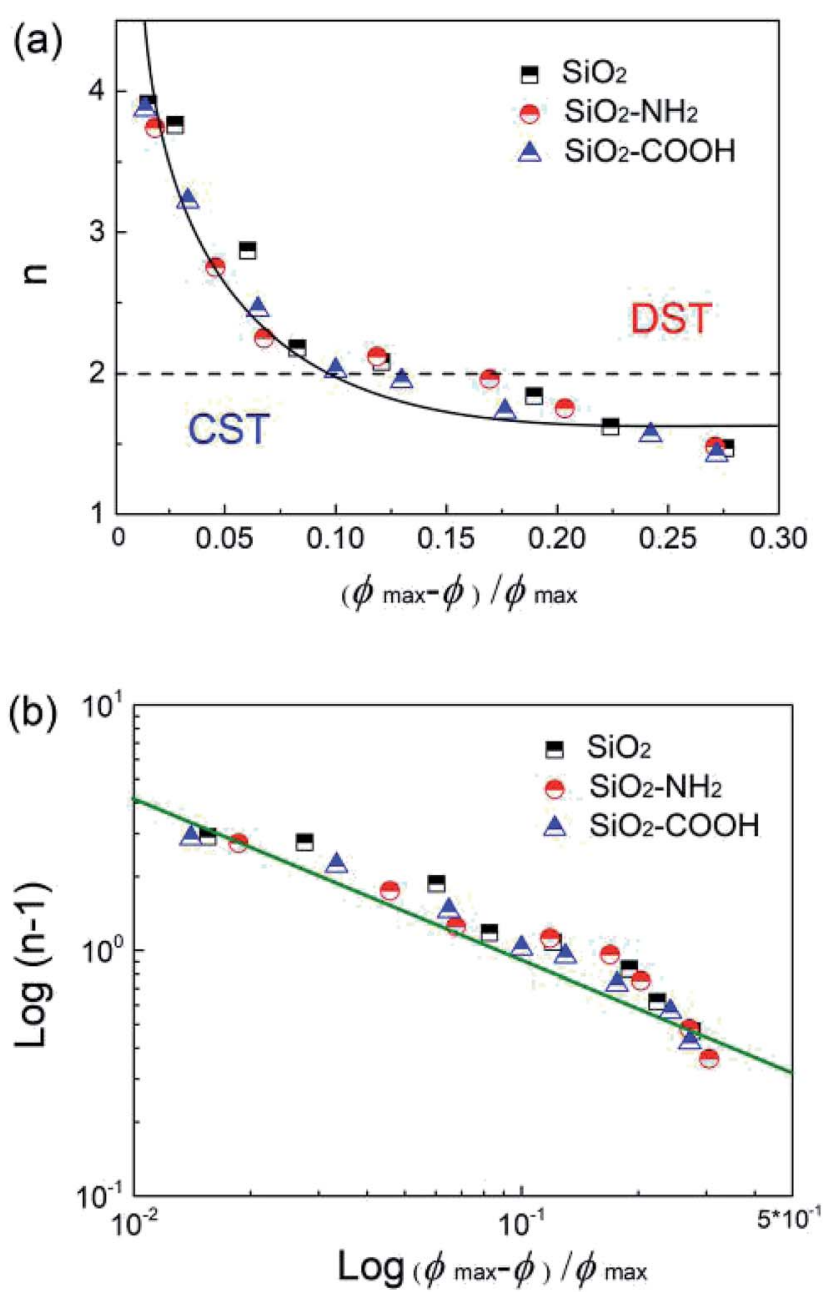

Fig. 10 The measured $\mu_{0}$ of three kinds of suspensions ( $\phi=53 \%$ ) at various $\mathrm{pH}$ conditions and their $\phi \mathrm{BL}_{\max }$ corresponding to the onset of DST. The volume fraction effect on the flow index $n$. (a) Oswald-De Waele exponent $n$ as a function of the reduced volume fraction (the $\phi_{\max }$ was set at $64 \%$, when passing this $\phi$, the suspension is hard to flow); (b) the same data in a log-log plot. The solid line is a power-law fit for $(n-1)$ versus reduced volume fraction. 
a function of a reduced volume fraction which does not depend on the surface chemical property. Furthermore, the exponential form of the same data was fitted in Fig. 10b, all the data disperse around the fitted line well, meaning an exponential relation of $n$ with the volume fraction change. This understanding is helpful to predict and adjust the thickening degree by controlling the volume fraction of the suspensions.

\section{Conclusion}

To investigate the influence of the surface properties of the dispersed particles on the shear thickening behavior of the suspensions and find a convenient method to adjust the character of shear thickening, organic chains with $\mathrm{COOH}$ and $\mathrm{NH}_{2}$ end groups were grafted into the surface of $\mathrm{SiO}_{2}$ microspheres. The suspensions were prepared by matching prepared $\mathrm{SiO}_{2}$, $\mathrm{SiO}_{2}-\mathrm{NH}_{2}$ and $\mathrm{SiO}_{2}-\mathrm{COOH}$ microspheres with a PEG medium, respectively, their rheological behavior was measured and compared in detail.

Obvious differences in the rheological behavior were observed for the three types of suspensions. By contrast, for the same $\phi, \dot{\gamma} \mathrm{SiO}_{2}<\dot{\gamma} \mathrm{SiO}_{2}-\mathrm{NH}_{2}<\dot{\gamma} \mathrm{SiO}_{2}-\mathrm{COOH}$ is always valid, and their gap becomes larger with increased $\phi$, while the maximum viscosity displays the reverse, all of which reveals the large effects caused by the changed surface character of the microspheres. Though the grafted $\mathrm{NH}_{2}$ and $\mathrm{COOH}$ groups could strongly interact with the PEG medium by the hydroxyl groups, the introduced organic chains still destroy the solvation layer on the surface of the $\mathrm{SiO}_{2}$ microsphere formed by hydrogen bond interactions between the $\mathrm{OH}$ groups, and the particles slight aggregation more easily occurs due to the interaction of the macromolecular chains, both factors are against the occurrence of shear thickening. Moreover, the suspensions with mixed $\mathrm{SiO}_{2}-\mathrm{NH}_{2}$ and $\mathrm{SiO}_{2}-\mathrm{COOH}$ particles show higher $\dot{\gamma}$, lower maximum viscosity and stronger shear thinning than that of suspensions with the single particle. This intensified weakening of thickening is attributed to the stronger aggregation tendency between the particles due to electron acceptor and donor characteristics the of $-\mathrm{NH}_{2}$ and $-\mathrm{COOH}$ groups, respectively.

For controlling the rheological behavior more effectively, a certain amount of aqueous solution with various $\mathrm{pH}$ values was added to the suspensions to adjust the interactions in the system. For the three kinds of suspensions, the effect of the $\mathrm{pH}$ value on their rheological behavior is different. For the $\mathrm{SiO}_{2}$ and $\mathrm{SiO}_{2}-\mathrm{NH}_{2}$ suspensions, the increased pH leads to a higher $\dot{\gamma}$ and larger maximum viscosity, the initial shear thinning becomes weaker and the shear thickening behavior is more abrupt. While the influence of the $\mathrm{pH}$ value on the $\mathrm{SiO}_{2}-\mathrm{COOH}$ suspensions is inverse. It fully proves that the rheological behavior of the suspensions could be adjusted by changing the micro-environment of the microspheres. The rule is summarized as follows: when the aqueous solution tends to combine with the microspheres, the shear thickening exhibits a greater weakening, whereas when the aqueous solution combines with the media more easily, the shear thinning exhibits a more obvious change, all of which is determined by the competing interaction between the components in the suspension.
Changing the mass of the added aqueous solution should only change the degree of the effect but not the original trend.

Based on the above test results, two theory models were used to analyze the related mechanism. Focusing on the start of shear thickening, the $\tau_{\mathrm{c}}$ was analyzed by using the Wagner model, in which, the measured zeta potential was used to stand for the surface potential of particles. It was found that the measured $\tau_{\mathrm{c}}$ and predicted $\tau_{\mathrm{c}}$ both maintain a similar tendency as that of the absolute value of the zeta potential which directly depends on the $\mathrm{pH}$ value, indicating that the interaction between the particles affects the onset of shear thickening significantly. Though the value of the measured and predicted $\tau_{\mathrm{c}}$ is similar, a gap between them still exists due to the more complex interactions in the actual system. Furthermore, friction theory was used to analyze the degree of shear thickening. Based on the calculated Sommerfeld number $s$ of this researched system, it could be inferred that the suspensions are in the "boundary lubrication" regime, in which the fixed coefficient of friction $\mu_{0}$ is independent of the load, speed and apparent contact area. Here, the independent friction coefficients $\mu_{0}$ of each particle at different $\mathrm{pH}$ was estimated using a designed similar friction test system. For each suspension with a special $\mu_{0}$, there exists a critical volume fraction $\left(\phi \mathrm{BL}_{\max }\right)$ corresponding to the onset of DST. With the increase of $\mu_{0}$, the $\phi \mathrm{BL}_{\max }$ decreases significantly, the rule is independent of the kind of particle in the suspension. This phenomenon reveals that $\mu_{0}$ is the key factor to determine the degree of shear thickening. Moreover, an exponential relationship between the decreased $\phi$ and $n-1$ ( $n$ is index $\tau \propto \gamma^{n}$ ) was found, indicating that the effect of $\phi$ on the thickening degree is reduced gradually with the decrease of $\phi$.

\section{Conflicts of interest}

There are no conflicts to declare.

\section{Acknowledgements}

We acknowledge the financial supports of the National Natural Science Foundation of China (11502077), The Key Research and Development Plan of Hunan (2016SK2077).

\section{Notes and references}

1 S. R. Waitukaitis and H. M. Jaeger, Nature, 2012, 487, 205209.

2 M. van Hecke, Nature, 2012, 487, 174-175.

3 O. E. Petel, S. Ouellet and J. Loiseau, Appl. Phys. Lett., 2013, 120, 064103.

4 R. L. Hoffman, J. Colloid Interface Sci., 1974, 46, 491-506.

5 R. L. Hoffman, J. Rheol., 1998, 42, 111-123.

6 E. Brown and H. M. Jaeger, J. Rheol., 2012, 56, 875-923.

7 A. Fall, N. Huang, F. Bertrand, G. Ovarlez and D. Bonn, Phys. Rev. Lett., 2008, 100, 018301.

8 N. J. Wagner and J. F. Brady, Phys. Today, 2009, 62, 27-32.

9 X. Cheng, J. H. McCoy, J. N. Israelachvili and I. Cohen, Science, 2011, 333, 1276-1279. 
10 W. Q. Jiang, Y. Q. Sun, Y. L. Xu, C. Peng, X. L. Gong and Z. Zhang, Rheol. Acta, 2010, 49, 1157-1163.

11 S. S. Shenoy and N. J. Wagner, Rheol. Acta, 2005, 44, 360-371. 12 J. Melrose and R. Ball, J. Rheol., 2004, 48, 961-978.

13 B. J. Maranzano and N. J. Wagner, J. Rheol., 2001, 45, 12051222.

14 B. J. Maranzano and N. J. Wagner, J. Chem. Phys., 2001, 114, 10514-10527.

15 J. Kaldasch, B. Senge and J. Laven, Rheol. Acta, 2008, 47, 319323.

16 J. Kaldasch and B. Senge, Colloid Polym. Sci., 2009, 287, 1481-1485.

17 C. B. Holmes, M. Fuchs and M. E. Cates, Europhys. Lett., 2003, 63, 240.

18 C. B. Holmes, M. E. Cates, M. Fuchs and P. Sollich, J. Rheol., 2005, 49, 237-269.

19 M. Sellitto and J. Kurchan, Phys. Rev. Lett., 2005, 95, 236001.

20 E. Brown and H. M. Jaeger, J. Rheol., 2010, 56, 875-923.

21 A. Fall, N. Huang, F. Bertrand, G. Ovarlez and D. Bonn, Phys. Rev. Lett., 2008, 100, 018301.

22 A. Fall, F. Bertrand, G. Ovarlez and D. Bonn, J. Rheol., 2012, 56, 575-591.

23 E. Brown and H. M. Jaeger, Rep. Prog. Phys., 2014, 77, 046602. 24 D. Bi, J. Zhang, B. Chakraborty and R. P. Behringer, Nature, 2011, 480, 355-358.

25 N. Y. C. Lin, B. M. Guy, M. Hermes, C. Ness, J. Sun, W. C. K. Poon and I. Cohen, Phys. Rev. Lett., 2015, 115, 228304.
26 B. M. Guy, M. Hermes and W. C. Poon, Phys. Rev. Lett., 2015, 115, 088304.

27 R. Mari, R. Seto, J. F. Morris and M. M. Denn, J. Rheol., 2014, 58, 1693-1724.

28 J. R. Royer, D. L. Blair and S. D. Hudson, Phys. Rev. Lett., 2016, 116, 188301.

29 R. Seto, R. Mari, J. F. Morris and M. M. Denn, Phys. Rev. Lett., 2013, 111, 218301.

30 N. Fernandez, R. Mani, D. Rinaldi, D. Kadau, M. Mosquet, H. Lombois-Burger, J. Cayer-Barrioz, H. J. Herrmann, N. D. Spencer and L. Isa, Phys. Rev. Lett., 2013, 111, 108301. 31 M. Wyart and M. E. Cates, Phys. Rev. Lett., 2014, 112, 098302. 32 E. Brown, N. A. Forman, C. S. Orellana, H. J. Zhang, B. W. Maynor, D. E. Betts, J. M. DeSimone and H. M. Jaeger, Nat. Mater., 2010, 9, 220-224.

33 S. Wang, W. Q. Jiang, W. F. Jiang, F. Ye, Y. Mao, S. H. Xuan and X. L. Gong, J. Mater. Chem. C, 2014, 34, 7133-7140.

34 T. Yokoi, Y. Sakamoto, O. Terasaki, Y. Kubota, T. Okubo and T. Tatsumi, J. Am. Chem. Soc., 2006, 128, 13664-13665.

35 J. Warren, S. Offenberger, H. Toghiani, C. U. Pittman Jr, T. E. Lacy and S. Kundu, ACS Appl. Mater. Interfaces, 2015, 7, 18650-18661.

36 T. Jiang and C. F. Zukoski, Macromolecules, 2012, 45, 97919803.

37 T. Glomann, A. Hamm, J. Allgaier, E. G. Hübner, A. Radulescu, B. Farago and G. J. Schneider, Soft Matter, 2013, 9, 10559-10571.

38 Y. S. Lee and N. J. Wagner, Rheol. Acta, 2003, 42, 199-208. 